\title{
Analysis on voltage characteristics of distribution network considering rolling mill load and Research on control measures
}

\author{
Aihua Wang ${ }^{1}$, Xiaotong Zhang ${ }^{2}$, Zhenyu Shi ${ }^{3}$, Shunjiang Wang ${ }^{1}$ and Dianyang $\mathrm{Li}^{1}$ \\ ${ }^{1}$ State Grid Liaoning Electric Power Co., Ltd., Shenyang 110000, China. \\ ${ }^{2}$ State Grid Liaoning Electric Power Co., Ltd. Electric Power Research Institute,Shenyang 110000, China. \\ ${ }^{3}$ School of Electrical Engineering, Northeast Electric Power University,Jilin 132012, China.
}

\begin{abstract}
In view of the voltage fluctuation and flicker of distribution network caused by rolling mill type typical load in some distribution networks and the reduction of power quality of power grid, it is necessary to analyze the voltage characteristics of distribution network, and adopt an effective governance measure to suppress harmonics in distribution network and improve power quality of power grid. The mathematical models of several typical loads corrected based on the measured data are established, and the influence radiation range of the corrected rolling mill load model on the distribution network voltage is analyzed, and a SVC (static var compensator) governance measure with SOGI phase-locked strategy is adopted for governance. Finally, combined with the actual operation parameters of a substation of State Grid, the power system simulation model is built by using simulation software, and the effectiveness of the governance measures adopted in this paper is verified by simulation analysis.
\end{abstract}

\section{Introduction}

At present, with the rapid development of economy in some regions of China, the power load is growing very rapidly, especially the impact load of rolling mill is developing towards the direction of large capacity and ultra-high power ${ }^{[1]}$. It is because of the rapid development of steel-making industry that the power grid has been affected by many rolling mill loads, resulting in power supply voltage fluctuations and power quality reduction in some distribution networks. At the same time, due to the lack of effective means of dynamic reactive power regulation, it has a certain impact on the power users in the distribution network with rolling mill load. This has also aroused great concern of power workers, and the modeling of rolling mill load and its effective governance measures are regarded as the top priority to improve the voltage level of distribution network ${ }^{[2]}$.

In foreign countries, because the industrial development of European and American countries is many years earlier than that of domestic countries, the power quality problems caused by rolling mill load to power grid have been paid attention to for a long time.

Therefore, a relatively perfect static var compensator (SVC) has been developed in foreign countries for a long time ${ }^{[3-5]}$. And the device is put into the distribution network with rolling mill load, so that the power quality in the distribution network can be effectively controlled.
In China, compared with developed countries, China started late in industry, and its technology is relatively backward, so in the initial stage of industrial construction, China only pays attention to output and ignores the impact of impact load on power quality of distribution network. It was not until the 1980s that China began to pay attention to this influence, and gradually began to study the field of static var compensation technology. Although in a short period of time, China's research on static var compensator has made remarkable achievements ${ }^{[6]}$, but from many aspects, there is still a certain gap between China's reactive power compensation technology and foreign companies ${ }^{[7]}$.

Combined with the current situation of distribution network with rolling mill load at home and abroad and the control measures of reactive power compensation, this paper first analyzes the working principle of rolling mill load, and combined with the measured data, establishes the rolling mill load model based on data correction. Then, combined with the influence of rolling mill load on distribution network voltage, a SVC governance measure with SOGI phase-locked strategy is adopted. Finally, the effectiveness of the strategy is verified by simulation analysis.

\section{Rolling mill load modeling based on data correction}

The drive of rolling mill in steel plant mainly includes asynchronous motor, synchronous motor and DC motor.

*Corresponding author's e-mail: 20162669@neepu.edu.cn 
The load modeling of rolling mill driven by different types should be based on the corresponding motor model. Considering the wide applicability of asynchronous motor model in power system load model, a new rolling mill model which can reflect the initiative of power demand based on data correction is established by taking asynchronous motor as an example with measured data.

In order to reflect the initiative and impact of rolling mill load, the load torque $T_{\mathrm{m}}$ in the dynamic model of asynchronous motor can be expressed as follows:

$$
T_{m}=F\left(T_{0}, T_{1}, T_{2}, T_{3}, T_{4} ; V_{m}\right)+K_{L}\left[\alpha+(1-\alpha)(1-S)^{N}\right]
$$

In the formula: $F\left(T_{0} \sim T_{4}, \Delta T_{\mathrm{m}}\right)$ is the change part of the load torque in the process of feeding and discharging; $K_{\mathrm{L}}\left[\alpha+(1-\alpha)(1-\mathrm{s})^{\mathrm{N}}\right]$ is the no-load or basic load torque of the mill, $T_{\mathrm{m} 0} ; s$ is the motor slip; $K_{L}$ is the load coefficient; $\alpha$ is the resistance torque coefficient related to the speed.

The load torque expression (1) not only reflects the demand of the load torque in the rolling mill production process, but also reflects the influence of the speed change on the actual load torque.

The rolling mill model reflecting the power demand initiative of impact load is established, as shown in formula (2):

$$
\left\{\begin{array}{l}
\frac{d_{s}}{d_{t}}=\frac{1}{T_{j}}\left(T_{m}-T_{e}\right) \\
\frac{d_{e}}{d_{t}}=\frac{1}{T^{\prime}{ }_{d 0}}\left[-e^{\prime}-j K_{Z}\left(X-X^{\prime}\right) I\right]-j 2 \pi s f_{0} e^{\prime} \\
T_{m}=F\left(T_{0}, T_{1}, T_{2}, T_{3}, T_{4} ; \Delta T_{m}\right)+K_{L}\left[\alpha+(1-\alpha)(1-s)^{N}\right] \\
T_{e}=-K_{P}\left(e_{R} I_{R}+e_{I} I_{I}\right) \\
e^{\prime}-j K_{Z} X^{\prime} I-U_{t}=0
\end{array}\right.
$$

In the formula: $T_{\mathrm{j}}$ is the inertia time constant of the motor; $T_{\mathrm{m}}$ is the torque of the load; $T_{\mathrm{e}}$ is the electromagnetic torque; $E$ ' is the equivalent internal potential of the induction motor; $T_{\mathrm{d}}$ ' 0 is the time constant of the rotor winding when the stator is open; $K_{Z}$ is the coefficient that converts the basic impedance of the motor itself into the basic impedance of the system; $X$ is the no-load reactance of the stator; $X^{\prime}$ is the short circuit reactance; $I$ is the stator current phasor; $f_{0}$ is the system frequency; $K_{P}$ is the conversion coefficient from the standard unit value of the system to the standard unit value of the motor itself; $e_{R}$ and $e_{I}$ are the real and imaginary parts of the internal potential; $I_{\mathrm{R}}$ and $I_{\mathrm{I}}$ are the real and imaginary parts of stator current; $U_{\mathrm{t}}$ is the phasor of generator terminal voltage.

\section{Modeling and control strategy of SVC}

\subsection{Mathematical model of SVC}

SVC device is mainly used for reactive power compensation of distribution network with rolling mill load. The capacitor in SVC is used to compensate the inductive reactive power, and the reactor is used to compensate the capacitive reactive power. By controlling the trigger angle $\alpha$, the compensation susceptance of power devices to the distribution network is adjusted, so as to change the amount of capacitive reactive power compensated by the reactor in the reactive power compensation, and then adjust the supply voltage of the distribution network ${ }^{[8]}$.

The equivalent fundamental susceptance in TCR can be expressed as follows:

$$
B_{T C R}(\alpha)=\frac{2(\pi-\alpha)+\operatorname{Sin} 2 \alpha}{\pi X_{L}}
$$

The equivalent susceptance of TCR $+\mathrm{FC}$ (Fixed capacitor) combined SVC is:

$$
B_{S V C}=-\left(\omega C-\frac{2(\pi-\alpha)+\operatorname{Sin} \alpha}{\pi \omega L}\right)
$$

According to the calculation, the reactive power absorbed by TCR from the system is as follows:

$$
Q_{T C R}=\frac{U^{2}}{X_{L}}=U^{2} \frac{2(\pi-\alpha)+\operatorname{Sin} \alpha}{\pi \omega L}
$$

When the capacitor is on, the reactive power injected into the power system is:

$$
Q_{F C}=U^{2} \omega C
$$

The reactive power injected into the system by the TCR+FC combined SVC is the algebraic sum of the reactive power injected into the system when the FC-type SVC and the TCR-type SVC are used alone, which can be obtained by equations (5) and (6):

$$
Q_{S V C}=U^{2}\left(\omega C-\frac{2(\pi-\alpha)+\operatorname{Sin} \alpha}{\pi \omega L}\right)
$$

In the formula: $\alpha$ is the control angle; $\omega$ is the rated angular velocity of the power supply, in $\mathrm{rad} / \mathrm{s} ; \mathrm{X}_{\mathrm{L}}=\omega L$ is the fundamental reactance of the reactor, in $\Omega ; C$ is the capacitance of the capacitor, in $\mathrm{F}$; $U$ is the bus voltage where the SVC is connected, The unit is $\mathrm{kV}$.

\subsection{Control strategy of SVC with SOGI phase locked strategy}

The TCR + FC SVC is composed of shunt capacitor bank and SCR controlled reactor. This type of SVC can track the fluctuating reactive power of power grid or load, and carry out random real-time compensation, so as to maintain the voltage stability of distribution network. The three-phase wiring diagram of TCR + FC SVC is shown in Figure 1.

According to the reactive power change of the actual power load $Q_{\mathrm{L}}$, adjust the reactive power change $Q_{\mathrm{TCR}}$ of the fundamental reactance. When $Q_{\mathrm{L}}$ becomes small, adjust the trigger angle to make $Q_{\mathrm{TCR}}$ increase. On the contrary, when QL becomes large, adjust the trigger angle to make $Q_{\mathrm{TCR}}$ decrease. In a word, adjust to ensure that the sum of the two numbers is constant, that is, $Q_{\mathrm{L}}+$ $Q_{\mathrm{TCR}}=\mathrm{K}, \mathrm{K}$ is constant. If $Q_{\mathrm{S}}=Q_{\mathrm{FC}}-\left(Q_{\mathrm{L}}-Q_{\mathrm{TCR}}\right)$, then $Q_{\mathrm{S}}$ represents the reactive power obtained from the grid. If $Q_{\mathrm{s}}$ is a constant or zero, then the power factor of the power grid will remain unchanged, and the voltage will remain unchanged, which will achieve the purpose of reactive power compensation, so as to restrain the voltage fluctuation and flicker caused by load changes. 
The variable reactor used to control $Q_{\mathrm{TCR}}$ is composed of reactor and two anti parallel thyristors in series. If we want to change the compensation capacity of the reactor, we need to control the trigger angle of the thyristor. In this way, we can not only control the current at both ends of the reactor, but also adjust the reactive power $Q_{\mathrm{TCR}}$ of the reactor by changing the current.

In this paper, the control thyristor trigger circuit is divided into two parts: synchronization and trigger. The first part is the PLL (phase locked loop) module. The angle $\theta$ is obtained by measuring the bus voltage. The angle $\theta$ changes continuously from $0^{\circ}$ to $360^{\circ}$ according to the input signal frequency. However, when the supply voltage of the distribution network fluctuates and flickers, a large number of negative sequence voltage will be generated in the distribution network, and the traditional PLL will no longer be able to achieve accurate phase locking. Therefore, in order to provide accurate phase-locked angle for SVC control, this paper adopts the phase-locked strategy with SOGI (the phase-locked loop control strategy based on SOGI can realize the positive and negative sequence separation of voltage, and separate the negative sequence voltage in the distribution network supply voltage). The schematic diagram of SOGI is shown in Figure2, in which two outputs are $Y(\mathrm{~s})$ and $H(\mathrm{~s})$, and the expressions of band-pass filter and low-pass filter can be obtained from the schematic diagram, as shown in formulas (8) and (9):

$$
\begin{gathered}
D(S)=\frac{Y(S)}{F(S)}=\frac{K \omega_{0} S}{S^{2}+K \omega_{0} S+\omega_{0}{ }^{2}} \\
Q(S)=\frac{H(S)}{F(S)}=\frac{K \omega_{0}{ }^{2}}{S^{2}+K \omega_{0} S+\omega_{0}{ }^{2}}
\end{gathered}
$$

Where $F(S)$ is the input voltage; $\omega_{0}$ is the undamped natural angular frequency; $K$ is the damping coefficient (when $\mathrm{K}=1.414$, the system has good stability and dynamic performance), and the damping coefficient in this paper is 1.414 .

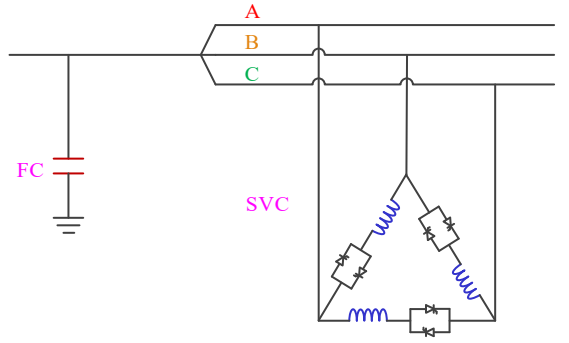

Figure1 Three phase wiring diagram of TCR + FC SVC

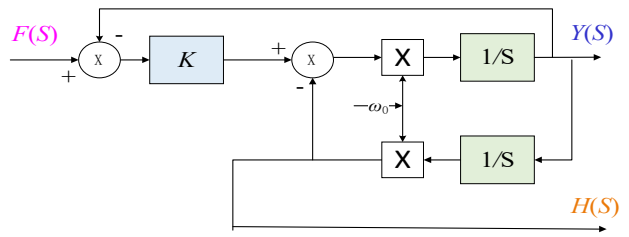

Figure2 Schematic diagram of SOGI

The second part is the trigger pulse generation module, because the system is a three-phase system, so the system uses a six pulse trigger module. In the system, the output signal $\theta$ of phase-locked loop SOGI-PLL) based on SOGI control strategy is the input signal of $\mathrm{H}$ in the module, and the $\mathrm{L}$ in the module is given by the trigger angle $\alpha_{1}$ of thyristor. In the simulation, when $\alpha_{1}$ intersects with the rising edge of signal $\theta$, trigger signal $\mathrm{P}$ will be generated. By determining the difference $\triangle B_{\mathrm{TCR}}$ between the compensation susceptance generated when the trigger angle is $\alpha_{1}$ and the compensation susceptance calculated after the actual measurement, the given trigger angle $\alpha_{1}$ is adjusted until $\triangle B_{\mathrm{TCR}}$ is close to 0 , and the trigger angle $\alpha$ is used as the trigger angle of SVC. Finally, when the load changes, the SVC compensation system is controlled to improve the supply voltage quality of the distribution network. The control principle is shown in Figure 3.

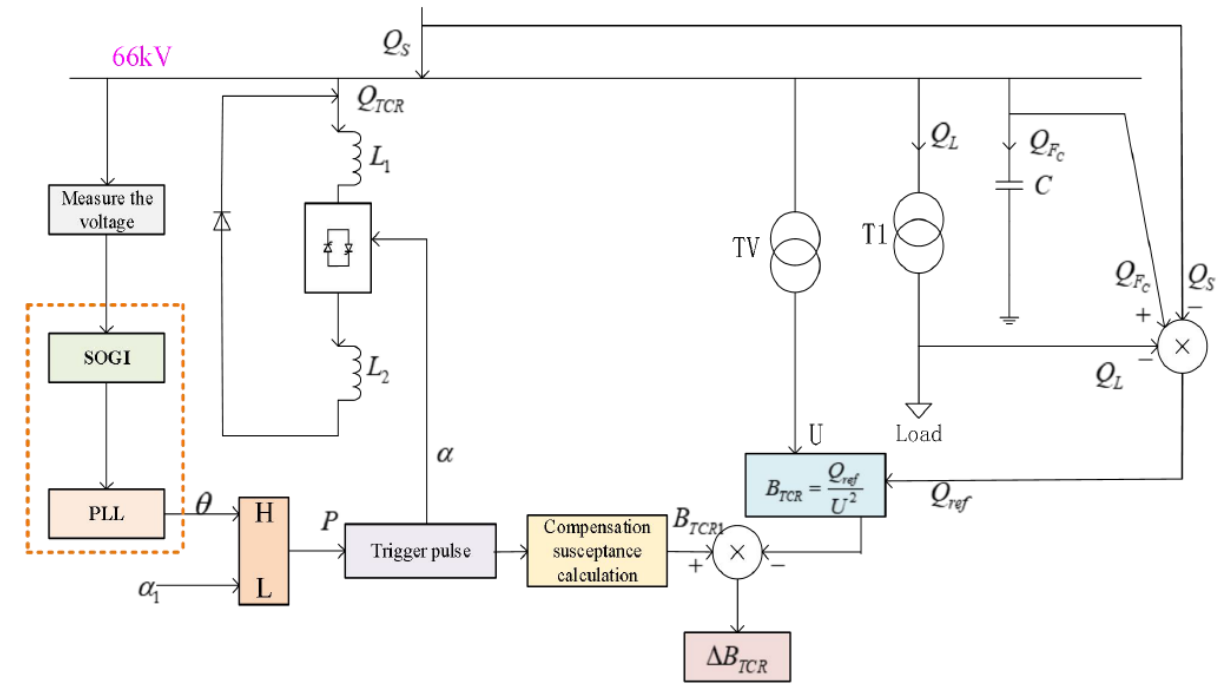

Figure3 Control principle of TCR + FC SVC with SOGI phase-locked strategy

\section{Simulation analysis and verification}

According to the load characteristics of rolling mill, combined with the actual operation of a substation of
State Grid, based on the theory and design methods of the previous chapters, a TCR + FC SVC with SOGI phase-locked strategy is designed at $66 \mathrm{kV}$. And through the simulation analysis, it further verifies that the 
governance measures adopted in this paper can improve the power quality of the distribution network.

\subsection{Voltage characteristic analysis of distribution network}

Figure4 is the equivalent diagram of the actual line of the power grid. The power grid connection mode described in the equivalent diagram is that $220 \mathrm{kV}$ is reduced to $66 \mathrm{kV}$ bus by transformer, and then to $10 \mathrm{kV}$ BUS3 and BUS4 by transformer. Constant power and constant impedance loads are connected to BUS4 via transformer T6. SVC reactive power compensation device is connected to $66 \mathrm{kV}$ BUS2, and rolling mill load is connected to BUS3 through transformer T5. In this paper, the influence of rolling mill load connected to the distribution network on the distribution network voltage is analyzed.

When the rolling mill load is $1.5 \mathrm{~s}$, it is connected to the distribution network through the transformer. Through the detection of the voltage at BUS2, BUS3 and BUS4, the influence of the rolling mill load on the voltage of the distribution network is further explained. In this paper, the voltage at BUS2 is $E_{1}$, the voltage at BUS4 is $E_{2}$, and the voltage at BUS3 is $E_{3}$. The simulation software is used to build the model of the power grid (the A-phase voltage is taken as an example for all the point voltages in this paper). The waveform of voltage instantaneous value per unit at each point before and after rolling mill load input is shown in Figure5.

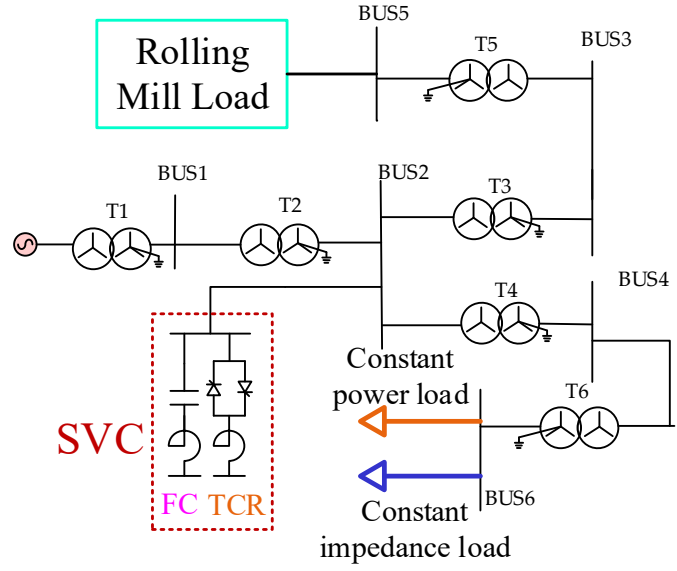

Figure4 Equivalent diagram of actual line of power grid

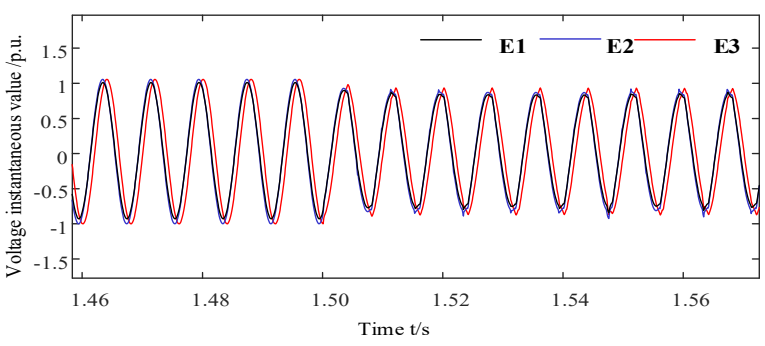

Figure 5 Voltage instantaneous value waveform under stand unit value

By comparing the magnitude and waveform of the voltage at each point when the rolling mill load is not connected to the distribution network and after it is connected to the distribution network, it can be seen that the rolling mill load connected to the distribution network will not only make the voltage of the distribution network under the line where the load is located drop and produce distortion, but also radiate to other branches in the distribution network, resulting in voltage reduction and distortion at other branches in the distribution network.

\subsection{Governance measures}

For a series of effects of rolling mill load on the voltage of the distribution network, the article will connect the TCR+FC type SVC governance measures without SOGI phase lock strategy and TCR+FC type SVC governance including SOGI phase lock strategy at BUS2. By comparing and analyzing the recovery of distribution network voltage under the two control measures, the effectiveness of the control measures adopted in this paper is verified.

In this paper, the above two treatment measures with a trigger angle of $145^{\circ}$ will be used at $2.5 \mathrm{~s}$. The voltage at each point of TCR+FC type SVC without SOGI phase locked strategy is shown in Figure6, and the voltage at each point of TCR+FC type SVC with SOGI phase locked strategy is shown in Figure 7.

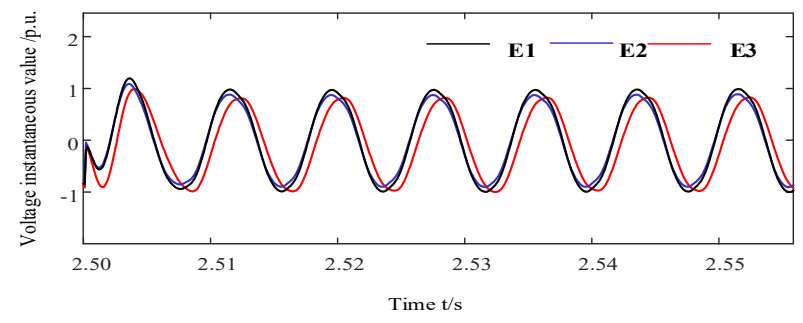

Figure 6 Voltage instantaneous value

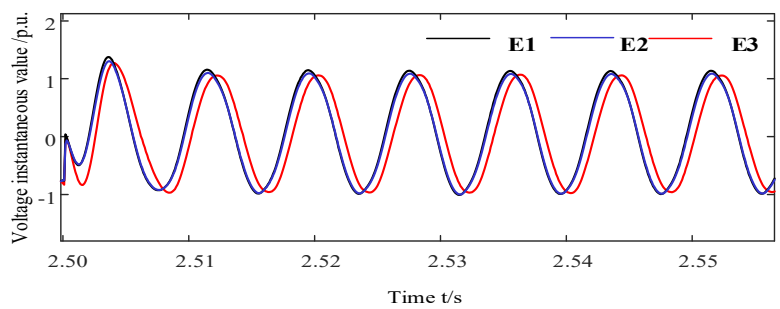

Figure 7 Voltage instantaneous value

Through comparative analysis, it can be seen that the SVC governance measures without sogi phase-locked strategy can improve the distribution network voltage and reduce the distribution network voltage distortion rate to a certain extent, but the effect is not obvious. The positive sequence voltage can be obtained through the positive and negative sequence separation of SOGI, and the positive sequence voltage can be sent to the PLL to achieve accurate phase locking. The improvement of phase-locked precision will promote SVC to manage the voltage of distribution network more effectively.

\section{Conclusion}

In this paper, based on the actual impact of rolling mill load on distribution network voltage, the simulation 
model is built by using simulation software, and three typical voltage nodes are selected as the key analysis in the model. By simulating the influence of rolling mill load connected to the power grid on the distribution network voltage and the control of two control measures on the distribution network voltage under $145^{\circ}$ trigger angle, the following conclusions are drawn:

(1) When the rolling mill load is connected to the distribution network, it not only has a serious impact on the voltage of the distribution network, but also has a huge impact on other branches, seriously reducing the power quality in the distribution network.

(2) Adopting TCR + FC type SVC governance measures with SOG Iphase-locked strategy can not only effectively adjust the voltage of each node in the distribution network, but also improve the power quality in the distribution network.

\section{References}

1. Li Zhiqiang, Han Zhiyong, Anning. Periodic impact load model reflecting the power demand initiative of rolling mill [J]. Power grid technology, 2011,35 (12): $72-76$

2. Liao Yantao, Hu Jun, Zhang Hailong. Time-varying Parameter Model of AC Electrical Arc Furnace for Power Quality Predictions and Analysis [J]. Electrical Engineering, 2016, 17(3): 41- 46.

3. B.K. Kumar, S.N. Singh,S.C. Srivastava. A decentralized nonlinear feedback controller for SVC with prescribed degree of stability for damping power system oscillations[P]. 7th IET International Conference on Advances in Power System Control, Operation and Management (APSCOM 2006),2006.

4. D.B. Kulkarni,G.R. Udupi. Optimized operation of SVC for minimal harmonics at distribution level[P]. IET-UK International Conference on Information and Communication Technology in Electrical Sciences (ICTES 2007), 2007.

5. H. Ebrahimirad, A. Choobdar,G.R. Dehnavi. A new control approach for voltage quality improvement in distribution power system by means of an SVC[P]. 4th IET International Conference on Power Electronics, Machines and Drives (PEMD 2008),2008.

6. Zhou Xiaohua, Zhang Yin, Liu Shengyong, et al. New Adaptive Dynamic Programming Voltage Control for Static Var Compensator[J]. Power System Protection and Control, 2018, 46(12): 77-84.

7. Fang Huanhuan, Shu Xinmei, Chen Bin. Development and prospect of medium voltage dynamic reactive power compensation device in China[J]. Electronic Technology Application, 2012, 38(8): 79-81

8. Sun Cong, Wang Yifan, Chen Guozhu, et al. Antiovercompensation control strategy of SVC device based on Steinmetz principle and instantaneous reactive power theory $[\mathrm{J}]$. Mechanical and Electrical Engineering, 2013, 30 (10): 1246-1249, 1272. 